\title{
Papers
}

\section{Doctors' emotional reactions to recent death of a patient: cross sectional study of hospital doctors}

\author{
Ellen M Redinbaugh, Amy M Sullivan, Susan D Block, Nina M Gadmer, Matthew Lakoma, Ann M \\ Mitchell, Deborah Seltzer, Jennifer Wolford, Robert M Arnold
}

\begin{abstract}
Objectives: To describe doctors' emotional reactions to the recent death of an "average" patient and to explore the effects of level of training on doctors' reactions.

Design: Cross sectional study using quantitative and qualitative data.

Setting: Two academic teaching hospitals in the United States.

Participants: 188 doctors (attending physicians (equivalent to UK consultants), residents (equivalent to UK senior house officers), and interns (equivalent to UK junior house officers)) who cared for 68 patients who died in the hospital.

Main outcome measures: Doctors' experiences in providing care, their emotional reactions to the patient's death, and their use of coping and social resources to manage their emotions.

Results: Most doctors (139/188, 74\%) reported satisfying experiences in caring for a dying patient. Doctors reported moderate levels of emotional impact (mean 4.7 (SD 2.4) on a 0-10 scale) from the death. Women and those doctors who had cared for the patient for a longer time experienced stronger emotional reactions. Level of training was not related to emotional reactions, but interns reported needing significantly more emotional support than attending physicians. Although most junior doctors discussed the patient's death with an attending physician, less than a quarter of interns and residents found senior teaching staff (attending physicians) to be the most helpful source of support.

Conclusions: Doctors who spend a longer time caring for their patients get to know them better but this also makes them more vulnerable to feelings of loss when these patients die. Medical teams may benefit from debriefing within the department to give junior doctors an opportunity to share emotional responses and reflect on the patient's death.
\end{abstract}

\section{Introduction}

Caring for dying patients is part of every doctor's clinical experience both during training and in subsequent practice. However, the literature on doctors' experiences in caring for the dying is sparse and mostly anecdotal. Many of these stories reflect the sadness, guilt, and stress caused by caring for dying patients. ${ }^{12}$ Such stress has been linked to professional burnout and may put doctors at risk of psychiatric disorders. ${ }^{3-5}$

Previous research has linked level of training with doctors' emotional reactions to deaths. In a longitudinal study "dealing with death and dying" was the most commonly reported source of stress among junior house officers, ${ }^{6}$ and it was related to psychological distress. ${ }^{7}$ As general practitioners, these same doctors did not find death and issues around dying to be stressful. ${ }^{8}$ These data provide some general expectations about emotional differences between junior hospital doctors and attending physicians (consultants). However, little has been published on junior doctors' use of coping strategies and social resources to manage powerful feelings evoked by their work.

We sought to extend previous research by investigating doctors' reactions to patients' deaths in two large US teaching hospitals. We explored the role of training level and other variables related to doctors and patients on doctors' experiences in caring for the dying. Finally, we examined how doctors coped with their emotions and the role of senior doctors in helping junior doctors to cope with their emotional reactions to patients' deaths.

\section{Methods \\ Participants}

Patient index cases

We obtained index cases by randomly reviewing charts of inpatients who died over the previous week and selecting one to two cases a week for inclusion in the study. Eligibility criteria for index patient cases included: a minimum hospital stay of 24 hours, death occurring on a general medicine unit or intensive care unit, and involvement of hospital doctors in the patient's care. Power calculations indicated that to detect group differences based on level of training we needed a minimum of 43 cases with $\beta=0.8$ and $\alpha=0.01$. Information abstracted from the charts of the 81 eligible cases included diagnosis, demographics, length of stay, whether or not the patient died in intensive care, and whether resuscitation was attempted.
Department of

Behavioural

Medicine and

Oncology,

University of

Pittsburgh Cancer

Institute, 5150

Centre Ave \#445,

Pittsburgh,

PA 15232, USA

Ellen M

Redinbaugh research instructor

Division of

Psychosocial

Oncology and

Palliative Care,

Dana-Farber

Cancer Institute

and Brigham and

Women's Hospital,

Department of

Psychiatry, Harvard

Medical School, 44

Binney St, Boston,

MA 02115, USA

Amy M Sullivan

assistant professor

Susan D Block

associate professor

Nina M Gadmer

project manager

Matthew Lakoma

research assistant

University of

Pittsburgh School

of Nursing, 415

Victoria Building,

200 Lothrop St,

Pittsburgh,

PA 15213, USA

Ann M Mitchell

assistant professor

Department of

Internal Medicine,

University of

Pittsburgh School

of Medicine, 200

Lothrop St,

Pittsburgh

Deborah Seltzer

research associate

Jennifer Wolford

research assistant

Robert M Arnold

professor

Correspondence to:

E M Redinbaugh

ellenr@pitt.edu

bmj.com 2003;327:185 


\section{Doctors}

We reviewed charts to identify the medical student, intern (junior house officer), resident (senior house officer), and primary attending physician (consultant) who were caring for the patient at the time of death. In the 81 index cases, only two medical students participated in an index patient's care and as they were both acting interns they were combined with the interns. Doctors who were providing temporary care while the patient's assigned doctor was off duty were ineligible for the study. Demographic comparisons of primary care physicians (similar to UK general practitioners) and attending physicians in intensive care and in general medicine indicated no significant differences between groups on demographic factors including age, sex, marital status, or race; therefore we combined all attending doctors into one group.

Analysis of variance comparisons of doctors from general medicine $(n=67)$, intensive care $(n=8)$, and primary care physicians $(\mathrm{n}=12)$ indicated that primary care physicians reported feeling closer to the patient $(F=3.58, \mathrm{P}<0.04)$, feeling more disturbed by the death $(F=4.50, \mathrm{P}<0.02)$, using more coping behaviours $(F=3.2, \quad \mathrm{P}<0.05)$, having more grief symptoms $(F=3.88, \mathrm{P}<0.03)$, and having more trauma symptoms on the impact of events scale $(F=4.17, \mathrm{P}=0.02)$. Doctors from general medicine and intensive care did not differ on any of the outcome variables. We reran our comparisons of interns, residents, and attending physicians excluding the 12 primary care physicians. None of the results changed, so we included primary care physicians in the group of attending physicians.

Of the 251 doctors who had cared for the 81 patients in the index cases, 246 remembered working with the patient and were therefore eligible for the study. Of the eligible doctors, 196 agreed to participate in the study ( $80 \%$ response rate). Eight were excluded because of insufficient data, resulting in a final sample of 188 doctors. Group comparisons of the participants (188/246 eligible doctors) and non-participants/ excluded doctors (58/246 eligible doctors) indicated that Jewish doctors were more likely to be nonparticipants/excluded doctors $\left(\chi^{2}=14.1, \mathrm{P}<0.01\right)$. The groups did not differ with regard to level of training, race, marital status, or age.

For a case to be included in data analyses we required a minimum of two interviewees. From the original 81 cases we excluded 13 because there was only one interviewee. There were no differences between included and excluded index cases with regard to diagnosis, demographics, length of stay, location of death, and resuscitation orders.

\section{Procedures}

We obtained informed consent from all participants before data collection. Qualitative data were gathered with a semistructured interview that allowed doctors to tell their individual stories about caring for a patient who died. At the end of the interview doctors were asked six questions that summarised the interview material (see table 1). Doctors then completed self administered questionnaires. We have focused on the quantitative data, including the six questions administered at the end of the interview and self administered questionnaires.

\section{Measures}

We grouped the measures under one of three headings: doctors' experiences in providing patient care, doctors' emotional reactions to the death of the patient, and doctors' use of coping resources after the patient's death (table 1). We used three measures that show good reliability and validity in general populations: the grief reaction scale, ${ }^{9}$ a short version of the impact of events scale, ${ }^{10}$ and the brief COPE. ${ }^{11}$ The distributions for these scales were positively skewed in our sample, indicating that most doctors endorsed either "never or rarely" or "occasionally" having a given symptom or using a particular coping strategy. Therefore we dichotomised the scale items into endorsed/ not endorsed and summed the rescaled items. The resulting distributions met normality assumptions necessary for conducting analyses.

We used literature review and discussions with experts to generate questions relevant to doctors' experiences in caring for the dying. We piloted and revised the questions to enhance their content validity. Questions generated by the study included: "How emotionally powerful was this death?" and "How satisfying was it to participate in the care of this patient?" Doctors' responses to the questions fell within a normal distribution.

\section{Data analyses}

Table 1 shows the outcome variables, including doctors' experiences, emotional reactions, and use of coping resources. The key predictor variable of interest was level of training (intern, resident, or attending physician); other predictors were variables related to doctors and patients, such as sex, marital status, duration of care (table 2). To reduce the probability of type I errors from multiple testing, we set a more stringent predetermined $\alpha$ of 0.01 (two tailed), and we have reported only those variables that were consistently related to outcome variables (that is, a given variable was significantly related to at least three outcome variables)

We report univariate statistics as means and standard deviations for continuous variables and percentages for categorical variables. We tested bivariate associations using $\chi^{2}$ tests to compare categorical variables, correlation coefficients to measure linear associations, independent groups $t$ tests to compare means, and analysis of variance to compare means of more than two groups.

The analysis of variance models to test the association between level of training and the outcome variables needed to account for doctors clustering within shared index patient cases. We used the CROSSTAB procedure (SUDAAN, Research Triangle Institute, NC) and SURVEYMEANS and SURVEYREG (SAS Institute, Cary, NC) procedures to compute the model estimates. ${ }^{12}$ These programs adjust for covariance related to cases among doctors' scores so that the three analysis of variance groups were independent of one another.

\section{Results}

\section{Doctors' experiences in providing patient care}

Most of the doctors $(154,82 \%)$ had not cared for the patient before the final admission to hospital. During 
Table 1 Summary of quantitative measures used to assess impact on doctor of death of patient

Measurement domain and description of scale Doctors' experience in providing patient care

Seven items assessed doctors' satisfaction with index patient's symptom management Items scored on 0 (least satisfied) to 10 (most satisfied) scale. Not all questions applicable (for example, management of conflict item not applicable if there was no conflict about patient care) to each patient so mean satisfaction score for each doctor was used in analyses

Four questions administered during interview. Each question scored on 0 (not at all) to 10 (extremely) scale. No cumulative scores

\section{Doctors' emotional reactions to patient's death}

Two questions administered during interview. Each question scored on a 0 (not at all) to 10 (extremely) scale.

Fourteen items from relative grief measurement scale ${ }^{9}$ that assessed doctors' grief response to index patient's death in week after death. Items scored 0 (rarely or never experienced) or 1 (occasionally, moderately often, or very often). Summed scores used range 0-14 (Cronbach's $\alpha=0.81$ )

Six items from impact of event scale ${ }^{10}$ that assessed doctors' intrusive thoughts and avoidant behaviour during week after patient's death. Items scored either 0 (rarely or never experienced) or 1 (occasionally, moderately often, or very often). Score range $0-6$ (Cronbach's $\alpha=0.73$ )

\section{Doctors' use of coping resources after the patient's death}

Brief situational COPE scale. ${ }^{11}$ Five coping items assessing how doctors coped with index patient's death during week after patient's death. Items scored 0 (rarely or never experienced) or 1 (occasionally, moderately often, or very often). Score range $0-5$ (Cronbach's $\alpha=0.64$ )

Two questions administered during interview. Each question scored on 0 (not at all) to 10 (extremely) scale

Fifteen yes/no questions that assessed people, other doctors, healthcare professionals, and significant others that doctor talked to about patient death and content of conversation (medical details and personal feelings)

One item where doctors identified person who was most helpful in providing doctor with social support

that admission, doctors knew the patient for only a short period of time: $68(36 \%)$ knew the patient for three days or less, another $68(36 \%)$ knew the patient for four to seven days, and only 53 (28\%) reported caring for the patient longer than a week. In total 117 doctors $(62 \%)$ classified their relationship to the

\section{What scale measures}

Variable name

Doctor satisfaction with: patient's pain control; patient's other physical symptom Satisfaction control; management of patient's emotional symptoms; management of patient's family distress; decisions regarding life prolonging care; management of conflict about patient's care; overall care provided to patient

How satisfying to participate in care of this patient How close was your relationship with this patient How much conflict regarding patient care How much did this patient suffer

How disturbing was this death

Disturb

How emotionally powerful was this death Powerful

Grief symptoms

Grief

Intrusive thoughts: pictures of patient pop into my mind; dreams about patient;

IES (impact of event scale)

avoidant behaviour: avoid getting upset when thinking about patient; avoid

dealing with feelings about patient's death; try not to think about patient's death; try not to talk about patient's death

Doctors' use of different coping strategies: try to see it in different, more positive light; find comfort in my spiritual or religious beliefs; get emotional Coping support from others; turn to work/other activities to take my mind off it; express my negative feelings

How much support from colleagues to cope with patient death How much support received from colleagues regarding patient death

Social resources doctors accessed to discuss index patient death

patient as not close (for example, $\leq 3$ on a $0-10$ point scale) (table 3). On the other hand, 21 (11\%) reported feeling very close to the patient, and this was associated with providing patient care for a longer period of time $(r=0.38, \mathrm{P}<0.01)$.

Table 2 Demographic characteristics of 68 patients and 188 doctors in cases in which patients died. Figures are numbers (percentage) of patients or doctors unless stated otherwise

\begin{tabular}{|c|c|c|c|c|c|}
\hline \multirow[b]{2}{*}{ Demographic variable } & \multirow[b]{2}{*}{ Index patients ( $\mathrm{n=68}$ ) } & \multicolumn{4}{|c|}{ Doctors } \\
\hline & & All $(\mathrm{n}=\mathbf{1 8 8})$ & Attending physician ( $\mathrm{n}=68$ ) & Residents ( $n=57$ ) & Interns ( $n=63)$ \\
\hline Mean (SD) age (years) & $65.0(18.2)$ & $33.5(7.7)$ & $41.8(6.9)$ & $29.4(2.8)$ & $28.7(3.2)$ \\
\hline Men & $36(53)$ & $122(65)$ & $57(84)$ & $30(53)$ & $36(57)$ \\
\hline Women & $32(47)$ & $66(35)$ & $11(16)$ & $27(47)$ & $27(43)$ \\
\hline \multicolumn{6}{|l|}{ Race: } \\
\hline White & $57(84)$ & $135(72)$ & $56(83)$ & $40(71)$ & $39(62)$ \\
\hline Non-white & $11(16)$ & $53(28)$ & $12(17)$ & $17(29)$ & $24(38)$ \\
\hline \multicolumn{6}{|l|}{ Marital status: } \\
\hline Married & $44(65)$ & $128(68)$ & $58(85)$ & $35(61)$ & $35(56)$ \\
\hline Not married & $24(35)$ & $60(32)$ & $10(15)$ & $22(39)$ & $28(44)$ \\
\hline \multicolumn{6}{|l|}{ Religion: } \\
\hline Catholic & $37(55)$ & $39(21)$ & $17(25)$ & $13(23)$ & $9(15)$ \\
\hline Protestant & $18(27)$ & $38(20)$ & $13(19)$ & $10(18)$ & $14(22)$ \\
\hline Jewish & $4(6)$ & $55(29)$ & $27(40)$ & $13(23)$ & $14(22)$ \\
\hline Other & $5(8)$ & $32(17)$ & $4(6)$ & $11(20)$ & $17(27)$ \\
\hline None & - & $24(13)$ & $6(9)$ & $9(16)$ & $9(15)$ \\
\hline Unknown & $3(4)$ & - & - & - & - \\
\hline \multicolumn{6}{|l|}{ Disease: } \\
\hline Cancer & $20(29)$ & - & - & - & - \\
\hline Other & $48(71)$ & - & - & - & - \\
\hline \multicolumn{6}{|l|}{ Location of death } \\
\hline Intensive care & $33(49)$ & - & - & - & - \\
\hline General medical & $35(51)$ & - & - & - & - \\
\hline Mean (SD) duration of care† & - & $2.8(1.0)$ & $2.9(1.2)$ & $2.8(0.9)$ & $2.8(1.0)$ \\
\hline
\end{tabular}

†Variable measured with ordinal categories: $1=$ cared for patient 1 day; $2=$ cared for patient 2-3 days; $3=$ cared for patient 4-7 days; $4=$ cared for patient 8-30 days; $5=$ cared for patient $1-6$ months; $6=$ cared for patient $6-12$ months; $7=$ cared for patient $1-3$ years 
Table 3 Responses of 188 doctors to death of patients measured by variables assessing experiences, emotional reactions, and use of coping resources. Figures are mean (SD) scores and numbers (percentage) of low, moderate, and high scorers

\begin{tabular}{|c|c|c|c|c|}
\hline & Mean (SD) & Low scorers* & Moderate scorers* & High scorers* \\
\hline \multicolumn{5}{|c|}{ Doctors' experiences in providing patient care (actual range) } \\
\hline Satisfaction (1-10)† & $7.0(1.7)$ & $7(4)$ & $70(38)$ & 107 (58) \\
\hline Satisfying $(0-10) \dagger$ & $5.8(2.6)$ & $48(26)$ & $51(27)$ & $87(47)$ \\
\hline Close $(0-9)$ & $3.1(2.3)$ & $117(62)$ & $51(27)$ & $21(11)$ \\
\hline Conflict $(0-10) \dagger$ & $2.9(2.9)$ & $123(67)$ & $29(16)$ & $31(17)$ \\
\hline Suffer $(0-10) \dagger$ & $3.6(2.4)$ & $95(52)$ & $64(35)$ & $24(13)$ \\
\hline \multicolumn{5}{|c|}{ Doctors' emotional reactions to patient's death (actual range) } \\
\hline Disturb $(0-10) \dagger$ & $3.9(2.6)$ & $102(55)$ & $41(22)$ & $43(23)$ \\
\hline Powerful $(0-10) \dagger$ & $4.7(2.4)$ & $67(36)$ & $62(33)$ & $58(31)$ \\
\hline Grief $(0-11) \ddagger$ & $2.3(2.7)$ & $135(75)$ & $34(19)$ & $11(6)$ \\
\hline Impact of event scale $(0-6) \S$ & $1.3(1.6)$ & $115(64)$ & $54(30)$ & $11(6)$ \\
\hline \multicolumn{5}{|c|}{ Doctors' use of coping resources after patient's death (actual range) } \\
\hline Coping (0-5)ף & $1.6(1.5)$ & $94(51)$ & $66(36)$ & $24(13)$ \\
\hline Support needed (0-9) & $1.7(2.1)$ & $154(83)$ & $24(13)$ & $8(4)$ \\
\hline Support received $(0-10) \dagger$ & $6.6(3.4)$ & $27(24)$ & $12(11)$ & $73(65)$ \\
\hline
\end{tabular}

${ }^{*}$ Ranges based on wording used for each measure.

†Low $=0-3$, moderate $=4-6$, high $=7-10$.

łLow $=0-3$, moderate $=4-7$, high $=8-11$.

$\S L$ ow $=0-1$, moderate $=2-4$, high $=5-6$

ILow $=0-1$, moderate $=2-3$, high $=4-5$.

One hundred and thirty nine (74\%) doctors thought that taking care of the patient was a satisfying or very satisfying experience (table 3). Longer durations of providing care were associated with more satisfying experiences $(r=0.20, \mathrm{P}<0.01)$. Few doctors reported conflict among family and members of the healthcare team regarding the goals of patient treatment $(32,17 \%)$, poor symptom management $(8$, $4 \%)$, or patient suffering $(24,13 \%)$.

Doctors' emotional reactions to the patient's death Descriptive analyses indicated that many doctors reported moderate emotional impact from a patient's death, though $58(31 \%)$ doctors rated the death as having strong emotional impact (table 3). Many doctors $(103,55 \%)$ reported that the patient's death disturbed them very little, but $43(23 \%)$ reported that the patient's death was very disturbing to them. On average, doctors reported experiencing two (out of a possible 14) symptoms of grief after the death. The most commonly reported symptoms were "feeling upset when thinking about the patient" $(88,47 \%)$ and feeling "numb" $(45,24 \%)$. Doctors reported around one symptom (out of six) on the impact of events scale, with "Avoid getting upset when thinking about the patient" being the most commonly endorsed item (70, $37 \%)$.

The differences in emotional reactions by level of training (from analysis of variance models) were non-significant. Although interns reported more symptoms of grief than attending physicians (mean 2.9 (SD 2.9) $v 1.7(2.5), \mathrm{P}<0.03)$, this finding was not significant. Longer durations of providing care were consistently associated with stronger emotional reactions: $r=0.21(\mathrm{P}<0.01)$ for emotional impact, $r=0.19$ $(\mathrm{P}<0.01)$ for disturbing, $r=0.26$, $(\mathrm{P}<0.01)$ for grief, and $r=0.20(\mathrm{P}<0.01)$ for the impact of events scale. Female doctors reported more symptoms of grief than male doctors (3.3 (3.2 $v 1.8(2.1), \mathrm{P}<0.01)$.

\section{Doctors' use of coping resources after the patient's} death

Doctors reported using an average of two coping strategies (out of five) to manage the emotions associ- ated with an index patient's death. The most commonly endorsed were "getting emotional support from others" $(86,46 \%)$, "trying to see the death in a different light to make it seem more positive" $(68,36 \%)$, and "turning to work or other activities to take your mind off of it" $(68,36 \%)$. Female doctors used more coping behaviours than male doctors (2.2 (1.5) v 1.4 (1.4), $\mathrm{P}<0.01$ ), and residents used more coping behaviours than either interns or attending physicians $(2.1$ (1.5) $v$ 1.6 (1.4) and $1.2(1.4), \mathrm{P}<0.01)$.

Most doctors $(156,83 \%)$ reported needing little emotional support from their colleagues (table 3). Interns reported needing more emotional support than attending physicians (2.3 (2.2) $v 1.1$ (1.7), $\mathrm{P}<0.01)$. Female doctors reported needing more emotional support than male doctors (2.5 (2.4) v 1.3 (1.7), $\mathrm{P}<0.01)$. Although most doctors in need of support felt they had received it from their colleagues, 66 (35\%) felt their needs had gone unmet.

Most residents (42, 74\%) and interns (43, 68\%) spoke to the attending physicians about the death of the patient (table 4$)$. However, residents $(48,84 \%)$ and interns $(56,89 \%)$ relied more on talking with each other or another resident about their experiences with the death (table 4). Less than a quarter of residents (14, $24 \%)$ and interns $(13,21 \%)$ identified an attending physicians as the most useful person in helping them cope with the patient's death (table 4).

\section{Discussion}

This study describes doctors' reactions to routine deaths of patients on general medicine and medical intensive care units in two teaching hospitals. The patients who died were usually new to the doctors, and most doctors did not feel close to them. Still, doctors reported moderate emotional impact from the patient's death and a substantial minority rated the death as being very disturbing. Doctors reported "feeling upset when thinking about the patient" more than any other grief symptom, and "getting emotional support from others" was their primary coping strategy. Contrary to our expectations, junior doctors did not 
Table 4 Social resources accessed by doctors and comparisons by level of training. Figures are numbers (percentage) of doctors

\begin{tabular}{|c|c|c|c|c|c|}
\hline & All $(n=182)$ & Attending physician $(n=64)$ & Residents ( $n=57)$ & Interns ( $\mathrm{n}=61)$ & $\chi^{2}$, P value \\
\hline $\begin{array}{l}\text { Did you talk to an attending } \\
\text { physician? }\end{array}$ & $118(65)$ & $34(53)$ & $42(74)$ & $42(68)$ & $6.76, P<0.05$ \\
\hline Did you talk to a resident? & $146(80)$ & $45(70)$ & $48(84)$ & $55(89)$ & $8.33, P<0.02$ \\
\hline Did you talk to a non-doctor HCP? & $95(52)$ & $35(55)$ & $29(51)$ & $31(50)$ & $0.11, P=0.95$ \\
\hline Did you talk to your spouse or SO? & $76(42)$ & $21(33)$ & $25(44)$ & $29(48)$ & $4.20, P=0.13$ \\
\hline \multicolumn{6}{|c|}{ Of the people listed above, who was most helpful?: } \\
\hline Attending physician & $38(21)$ & $11(17)$ & $13(24)$ & $13(21)$ & \multirow{5}{*}{$32.0, P<0.01$} \\
\hline Resident & $44(24)$ & $6(9)$ & $15(27)$ & $22(36)$ & \\
\hline Non-doctor HCP & $11(6)$ & $6(9)$ & $2(4)$ & $3(5)$ & \\
\hline Spouse or SO & $45(25)$ & $12(19)$ & $15(27)$ & $17(28)$ & \\
\hline No one & $44(24)$ & $29(46)$ & $10(18)$ & $6(10)$ & \\
\hline
\end{tabular}

$\mathrm{HCP}=$ healthcare professional; $\mathrm{SO}=$ significant other.

differ from attending physicians in their emotional reactions to patients' deaths. However, their need for and use of social support differed from that of attending physicians.

While all groups reported needing relatively low levels of support, interns reported the greatest need for emotional support, and they typically sought support from residents. Although interns and residents were usually successful in identifying someone among their peers who they could talk to about the death, it is striking that more than a quarter of interns and residents had no postmortem follow up with an attending physician.

Our findings regarding sex differences are consistent with broader psychological findings. Compared with men, women consistently report more psychological symptoms, ${ }^{13}$ greater need for social support, and higher levels of received support. ${ }^{14}$ Female doctors report more psychological distress than male doctors, whether they are interns, residents, or attending physicians. ${ }^{815-17}$ In our study, female doctors reported more symptoms of grief and used more coping resources than male doctors. In particular, female doctors needed and received more support from their colleagues than male doctors.

The amount of time a doctor spends taking care of a dying patient seems to be both a source of satisfaction and a source of distress. Longer duration of care was associated with a more satisfying experience and greater feelings of closeness to the patient. However, it was also associated with finding the death more disturbing and emotionally powerful as well as more reported symptoms of grief and trauma. Our findings are similar to qualitative research in which doctors describe satisfaction in providing good end of life care while simultaneously expressing a sense of loss after a patient they knew well died. ${ }^{18}$

\section{Potential risk factors}

Our data show that while most deaths do not elicit a strong emotional response in doctors, we can identify those situations in which greater needs for emotional support may be present. These include the presence of a long doctor-patient relationship or the involvement of a female doctor or an intern. Being able to identify deaths in which the clinicians may be "at risk" of higher levels of emotional distress provides educators with some guidance about situations in which extra attention to these issues is essential. Unfortunately, in our current environment, these needs are likely to be unaddressed. Research has shown that senior physi- cians in charge of training house officers tend to underestimate emotional distress in junior doctors. ${ }^{19} 20$ Furthermore, the culture of medicine, through its emphasis on biomedical issues and its lack of emotional support, ${ }^{21}$ may also contribute to trainees' reluctance to report needs for emotional support. Although interns and residents were usually successful in identifying someone among their peers to talk to about the death, 46\% (36) of attending physicians found that no one was helpful. What is the cumulative impact of emotionally draining deaths on attending physicians who "do not need" emotional support?

\section{Limitations}

Our study had several limitations. While we made every effort to contact respondents within two weeks of the patient's death, interviews were sometimes delayed, leading to potential recall bias. Although we used standardised instruments whenever possible, few standardised measures exist that specifically assess doctors' emotions. The quantitative measures used may have lacked the sensitivity to measure subtle and highly individual reactions to death that may be better elucidated through qualitative methods. We will report on our qualitative findings in subsequent papers.

\section{Conclusions}

Our research provides new insights into the effect of patients' deaths on doctors and raises some questions about current medical training in the United States. Doctors are moved by the deaths of the strangers for whom they care, and they are often powerfully affected by the deaths of patients with whom they have forged close relationships. The attending physicians in charge of the learning of their interns and residents do not often discuss these strong emotional responses. This conveys a message about how death is to be handled and potentially isolates learners who could benefit from having an opportunity to receive a seasoned perspective on what it is like to care for a patient who dies. A conspiracy of silence toward emotions can potentially cause trainees to develop maladaptive coping patterns that lead to burnout and other forms of emotional distress. ${ }^{22}$ The finding that a substantial number of trainees did not talk to their attending physician, and that most did not find attending physicians to be the most helpful resource in dealing with the death, points to a major gap in the clinical education of interns and residents and an important opportunity for attending physicians to improve their clinical training skills. As the clinical leader and role model for the 


\section{What is already known on this topic}

The mostly anecdotal evidence on caring for dying patients contains stories of sadness, guilt, and loss

Longitudinal data suggest that junior doctors may be more strongly affected by patients' deaths than senior doctors

Female doctors report more psychological distress than male doctors

\section{What this study adds}

Doctors are moved by the deaths of the strangers for whom they care

The amount of time a doctor spends taking care of a dying patient can be a source of both satisfaction and distress

Female doctors and interns may require more emotional support after patients' deaths

team, it is incumbent on the attending doctor to teach and model both excellent patient care and appropriate self care in managing challenging patients. Senior physicians have a major role in legitimising discussions of these issues and in helping doctors in training to construct their losses in a meaningful and adaptive manner. Further research should investigate both how the attending physicians respond to distress in junior doctors as well as studies of how junior doctors' opinions regarding how attending physicians can be supportive.

We thank Margaret McDonald and Pat Ruopp for their important contribution to the research project on which our paper is based.

Contributors: EMR was responsible for conceptualisation and design of the study, collection of the data, analysis and interpretation of data, and drafting of the manuscript. AMS analysed and interpreted the data, revised the manuscript, and advised on statistical analyses. SDB was responsible for conceptualisation and design of the study, wrote the grant application, oversaw data collection, analysed and interpreted the data, and revised and finally approved the manuscript. NMG was responsible for conceptualisation and design of the study, collected the data, analysed and interpreted the data, and revised the manuscript. ML analysed and interpreted the data, and revised the manuscript. AMM analysed and interpreted the data and revised the manuscript. DS analysed and interpreted the data and revised the manuscript. JW collected the data, analysed and interpreted the data, and revised the manuscript. RMA was responsible for conceptualisation and design of the study, wrote the grant application, oversaw data collection, analysed and interpreted the data, revised the manuscript, and finally approved the manuscript (in conjunction with SDB). EMR, RMA, and SDB are the guarantors.

Funding: Nathan Cummings Foundation. The guarantors accept full responsibility for the conduct of the study, had access to the data, and controlled the decision to publish.

Competing interests: None declared.

Ethical approval: The study received approval by the internal review board at each study site.

Meier DE, Back AL, Morrison RS. The inner life of doctors and care of the seriously ill. JAMA 2001;286:3007-14.

2 Mulder J. Transforming experience into wisdom: healing amidst suffering. J Palliat Care 2000;16:25-9.

3 Krakowski AJ. Stress and the practice of medicine. II. Stressors, stresses, and strains. Psychother Psychosom 1982:38:11-23.

4 Molassiotis A, van den Akker OBA. Psychological distress in nursing and medical staff on bone marrow transplant units. Bone Marrow Transplant $1995 ; 15: 449-54$

5 Whippen DA, Canellos GP. Burnout syndrome in the practice of oncology: results of a random survey of 1000 oncologists. J Clin Oncol 1991;2:1916-20

6 Firth-Cozens J, Morrison L. Sources of stress and ways of coping in junior house officers. Stress Medicine 1989;5:121-6.

Firth-Cozens J. Emotional distress in junior house officers. BMJ 1987;295:533-6.

8 Firth-Cozens J. Interventions to improve doctors' well being and patient care. Soc Sci Med 2001;52:215-22.

9 Jacobs SC, Kasl S, Ostfeld AM, Berkman L, Kosten TR, Charpentier P. The measurement of grief. Hospice Journal 1986;2:21-36.

10 Horowitz M, Wilner N, Alvarez W. Impact of events scale: a measure of Horowitz M, Wilner N, Alvarez W. Impact of eve
subjective stress. Psychosom Med 1979;41,209-18.

11 Carver CS. You want to measure coping but your protocol's too long: consider the brief COPE. Int J Behav Med 1997;4:92-100.

12 Lee EU, Forthofer RN, Lorimer RJ. Analyzing complex survey data. Vol 71 Thousand Oaks, CA: Sage Publications, 1989.

13 Whitfield KE, Clark R, Weidner G, Anderson NB. Sociodemographic diversity and behavioral medicine. J Consult Clin Psychol 2002;70:463-81.

14 Belle D. Gender differences in the social moderators of stress. In: Monat A, Lazarus RA, eds. Stress and coping: an anthology. 3rd ed. New York: A, Lazarus RA, eds. Stress and coping: an

15 Behnke M, Reiss J, Neimeyer G, Bandstra ES. Grief responses of pediatric house officers to a patient's death. Death Studies 1987;11:169-76.

16 Revicki DA, Whitley TW, Gallery ME. Organizational characteristics, perRevicki DA, Whitley TW, Gallery ME. Organizational characteristics, per-
ceived work stress, and depression in emergency medicine residents. Behav Med 1993;19:74-81

17 Graham J, Ramirez AJ, Cull A, Finlay I, Hoy A, Richards MA. Job stress and satisfaction among palliative physicians. J Palliat Med 1996;10:18594.

8 Field D. Special not different: General practitioners' accounts of their care of dying people. Soc Sci Med 1998;46:111-20.

19 Purdy RR, Lemkau JP, Rafferty JP, Rudiskill JR. Resident doctors in family practice: who's burned out and who knows? Fam Med 1987;19:203-8.

20 Urbach JR, Levenson JL, Harbison JW. Perceptions of house staff stress and dysfunction within the academic medical center. Psychiatr $Q$ and dysfunction

21 Papadatou D, Bellali T, Papazoglou I, Petraki D. Greek nurse and doctor grief as a result of caring for children dying of cancer. Pediatric Nursing 2002;28:345-53.

22 Association of Professors of Medicine. The well-being of doctors. Am J Med 2003;114:513-9.

(Accepted 30 June 2003) 